\title{
Reply to "Comment on 'EM induction in elongated conductors normal to a coastline with application to geomagnetic measurements in Nigeria' by J. Chen, H. W. Dosso, and S. Kang"
}

\author{
H. W. Dosso and A. K. Agarwal \\ Department of Physics and Astronomy, University of Victoria, Victoria, B.C., Canada V8W 3P6
}

(Received August 4, 1999; Accepted September 24, 1999)

Dr. P. F. Chen (hereafter PFC) has questioned two main differences between the CDK analogue model and his FDM numerical results: 1) The CDK analogue model in-phase $V_{y}$ fall off rapidly with increasing period, whereas his numerical $H_{z} / H_{y}$ do not; 2) The CDK quadrature $V_{y}$ do not show a reversal, whereas his $H_{z} / H_{y}$ do. Upon examining the curves in Fig. 4 carefully, it can be concluded that the CDK quadrature $V_{y}$ do, in fact, also indicate a reversal. It can clearly be seen from the shape of the CDK curves (dashed line), that with decreasing period each response curve is approaching a reversal, though at a period some what below $1 \mathrm{~min}$, being the lowest period included in the analogue model measurements. The CDK in-phase response curves as well, are each approaching a maximum at periods well below $1 \mathrm{~min}$, and should each reach a maximum at roughly the same period as the corresponding quadrature reversal, being characteristic of the specific location $(y)$ between the conductors. These shifts to longer periods with distance indicated in the CDK results, are similar to those in the PFC (solid line) results which for sites at increasing distances from the major conductor $(b)$, show shifts to longer periods to be roughly $T_{c}=2,3$, and 8 min at $y=-45,-40$, and $-30 \mathrm{~km}$ respectively. With respect to the more rapid fall-off in-phase response with increasing period, as well as the quadrature reversals at lower periods in the CDK 3D results as compared with the PFC results, these are precisely the characteristics that distinguish $3 \mathrm{D}$ from $2 \mathrm{D}$ responses. The CDK model for the $1-60 \mathrm{~min}$ period range is certainly $3 \mathrm{D}$ for all but the shortest periods, since for the near-end $(x=30 \mathrm{~km})$ traverse the conductor length is definitely too small to satisfy the criteron for a $2 \mathrm{D}$ conductor in terms of host conductivity skin depths. Thus, the PFC roughly constant in-phase $H_{z} / H_{y}$ over the 1-60 min range appear to show a $2 \mathrm{D}$ rather than what should be a $3 \mathrm{D}$ response.

Unfortunately, PFC does not include any explanation regarding the numerical grid design, the boundary conditions, or the convergence criteria used in his finite difference numerical model for the high conductivity contrast $(\sim 5000)$, and the large (1-60 $\mathrm{min}$ ) period range for which the highly resistive host skin depth changes by nearly a factor of 8 .

Copy right (C) The Society of Geomagnetism and Earth, Planetary and Space Sciences (SGEPSS); The Seismological Society of Japan; The Volcanological Society of Japan; The Geodetic Society of Japan; The Japanese Society for Planetary Sciences.
Did he use air layers above the surface, or did he use a surface boundary condition? If he used air layers, what was the height extent? Did he use the same uniform grids for all periods, or did he change the grids for different periods where appropriate in order to enhance the accuracy? Without stating any convergence criteria, he does state that his iteration did converge, though slowly, but does this necessarily constitute proof that the numerical results are valid? If the differences between the PFC and the CDK results cannot be attributed to numerical inaccuracies in the $\mathrm{PFC}$ results due to possible difficulties with grid design, boundary conditions, convergence, etc., then the departure from the observed CDK $3 \mathrm{D}$ results might only be attributed to basic differences in $V_{y}$ and $H_{z} / H_{y}$ responses for a $3 \mathrm{D}$ structure. Then the question remaining is, why for clearly a 3D model for which the analogue model $V_{y}$ show a $3 \mathrm{D}$ response, do the numerical $H_{z} / H_{y}$ show a $2 \mathrm{D}$ like response? Addressing this question might have been of interest to a broader readership than the one of attempting to prove the analogue model results to be in error.

Now the question arises as to why the PFC numerical results behave more like those of a 2D model for the CDK 3D model even at the longer periods? Is it perhaps, that the FDM technique encounters difficulties with a large conductivity contrast (5000 used here), as is sometimes experienced in numerical modelling? Has a difficulty already arisen in obtaining the Fig. 2 respnses, since if the dimensions (Fig. 1) are taken to be valid for $2 \mathrm{D}$ at $1 \mathrm{~min}$ period, then the two response curves in each of Figs. $2 \mathrm{a}$ and $2 \mathrm{~b}$ should be the same. This is not the case for the quadrature results $\left(H_{z} / H_{y}\right.$ and $V)$, since at a short distance from the conductor boundary the FDM 3D responses (dotted lines) over the host are about $40 \%$ larger and fall off much more slowly with range than the $2 \mathrm{D}$ responses (solid lines). This trend is opposite to that expected, since the end effects of a 3D structure should, if any thing, reduce the magnitude of the responses while increasing the fall-off with range compared with that for a $2 \mathrm{D}$ structure. This significant discrepency in the Fig. 2 results would appear to cast doubt on the validity of the PFC $H_{z} / H_{y}$ responses for the CDK 3D model.

The relatively constant FDM (Figs. 3 and 4) in-phase $H_{z} / H_{y}$ are very similar to the $2 \mathrm{D}$-like responses seen in the lower period range (Figs. 2 and 3) in the Chen and Dosso 
(1997) analogue model study of a central traverse over a $500 \mathrm{~km}$ length conductor at various distances from a straight ocean coastline. As in the case for the CDK model, the conductive basement was at a $200 \mathrm{~km}$ depth. In this work, the $T_{c}$ was between 2 and $3 \mathrm{~min}$ at the site nearest the major conductor (ocean) and was shifted to longer periods at more distant sites. Thus, the PFC (Figs. 3 and 4) relatively constant in-phase responses, as well as the $T_{c}$ values, are very similar to those in the Chen and Dosso (1997) study. It should be emphasised, that this observed similarity is contrary to what should be the case, since the responses at short periods for the central traverse over the $500 \mathrm{~km}$ length conductor are those of a 2D structure, while the FDM responses for the near-end $(x=30 \mathrm{~km})$ traverse in the CDK model should be those of a 3D structure. In a more recent work, Dosso et al. (1999) carried out an analogue model study of an elongated conductor for a range of conductor lengths, depths of burial, and conductive basement depths for both central and near-end traverses. Both $T_{c}$ shifts to shorter periods and more rapid response fall-off with period were seen for decreasing conductor lengths (which effectively transformed the conductor into a more 3D-like sructure at a given period). These analogue model results all cast further doubt on the validity of the FDM technique for providing the correct responses for complex 3D structures.

In numerical studies, an example of relatively constant inphase responses (similar to those shown in the PFC results in Fig. 4) over a moderate period range can be found in the Agarwal and Dosso (1990) 2D numerical study of a conductive plate for two conductive basement depths. In addition, a recent 3D numerical study by Agarwal and Dosso (1999) of a conductive block further confirms both the more rapid response fall-off with period, as well as the lower $T_{c}$ for $3 \mathrm{D}$, as compared with those for $2 \mathrm{D}$ structures. This latter numer- ical study uses the finite difference staggered grid algorithm of Agarwal et al. (1996) based on the fixed grid 3D numerical algorithm of Weaver et al. (1999). Both algorithms use surface boundary conditions and handle high conductivity contrasts successfully. These two numerical studies, as well as the analogue model studies discussed in the preceeding paragraphs, support the view that the PFC numerical FDM $H_{z} / H_{y}$ results do not follow the expected general behaviour of the 3D responses. Perhaps, the Fig. 7 model in the CDK (1997) work would be more appropriate for testing the FDM technique, since both $V_{x}$ and $V_{y}$ are provided for what definitely is a $3 \mathrm{D}$ structure.

\section{References}

Agarwal, A. K. and H. W. Dosso, On the behaviour of the induction arrows over a buried conductive plate-a numerical model study, Phys. Earth Planet. Inter., 60, 265-275, 1990.

Agarwal, A. K. and H. W. Dosso, EM induction over a large conductive block-a 3D numerical model study, presented at IUGG99, Birmingham, 1999.

Agarwal, A. K., X. H. Pu, and J. T. Weaver, Three-dimensional modelling with the magnetic field: are staggered grids the only way?, presented at the 13th workshop on electromagnetic induction in the earth, Hokkaido, Japan, 1996.

Chen, J. and H. W. Dosso, EM responses of an elongated conductor near an ocean-analogue model studies, Phys. Earth Planet. Inter, 99, 83-89, 1997.

Dosso, H. W., J. Chen, and Z. W. Meng, An analogue model study of the EM induction responses of elongated conductors (in preparation).

Weaver, J. T., A. K. Agarwal, and X. H. Pu, Three-dimensional finitedifference modelling of the magnetic field in geo-electromagnetic induction, in Three Dimensional Electromagnetics, edited by M. J. Oristaglio and B. R. Spiess, pp. 426-433, Geophysical Developments Series 7, Soc. Explor. Geophys., Tulsa, 1999.

H. W. Dosso (e-mail: hdosso@uvic.ca) and A. K. Agarwal 